\title{
Interfacial wetting in the q-state Potts model
}

\author{
B Derrida and $M$ Schick $\dagger$ \\ Service de Physique Theorique, CEN-Saclay, 91191 Gif-sur-Yvette Cedex, France
}

Received 21 June 1985, in final form 19 September 1985

\begin{abstract}
We consider an interface between two ordered phases of a $q$-state Potts model below its bulk first-order transition temperature. The mean-field equations are solved analytically for small $q-2$ and the solution shows that the interface is wetted by the disordered phase as the transition is approached. The excess absorption and surface entropy diverge logarithmically. Numerical calculations indicate this wetting also occurs at larger $q$.
\end{abstract}

\section{Introduction}

The behaviour of an interface between two phases as coexistence with a third phase is approached is the subject of current experimental and theoretical investigation (Sullivan and Telo Da Gama 1985). Attention centres on the question of whether a film of the third phase intervenes between the first two phases and, if so, whether its thickness is microscopic or macroscopic. In the latter case, the third phase is said to wet the interface. Examples include the wetting of the vapour/A-rich liquid interface by B-rich liquid in binary-liquid mixtures (Sullivan and Telo Da Gama 1985, Tarazona et al 1983), and the surface melting of a solid in coexistence with its vapour (Frenken and van der Veen 1985). The same question can be posed in systems in which there are more than three possible phases and has recently been studied in two-dimensional systems (Selke 1984, Yeomans and Derrida 1985). In particular, interfaces in the Potts model, the chiral Potts model and the Blume-Emery-Griffiths model were investigated. While the results of the last two were clear and understood (Selke 1984, Yeomans and Derrida 1985), those of the first in which the bulk transitions were first order were neither (Selke 1984). The interface between two ordered phases A and B in the Potts model might be thought to be simply due to the symmetry which exists between its ordered phases. In particular, this symmetry implies that the surface tension between any two ordered phases must be the same. An immediate consequence is that the A/B interface cannot be wetted by another ordered phase $\mathrm{C}$, but only by the disordered phase, if at all. This is in contrast with the chiral Potts model in which the Potts symmetry is broken so that there is more than one surface tension between ordered phases (Huse et al 1983). Further, as there is only one parameter in the model, the interaction strength $J$, the ordered and disordered phases can coexist only at a single temperature, the bulk transition temperature $T_{0}$. Thus, at this temperature, either the disordered phase wets the A/B interface or it does not. The parameter space is not large enough to permit a line of three-phase coexistence, as occurs in the Blume-EmeryGriffiths model or in binary-liquid mixtures, or the possibility of a wetting transition. It is the purpose of this paper to determine, within the context of mean-field theory,

† Permanent address: Department of Physics, University of Washington, Seattle, WA 98195, USA.

$0305-4470 / 86 / 081439+10 \$ 02.50$ (C) 1986 The Institute of Physics 
which of these two behaviours actually occurs. As emphasised by Widom (1977), this can only be done by solving the full set of $q-1$ coupled equations for the $q-1$ independent densities. In particular, one cannot appeal to effective one-density theories because such theories presume one behaviour or the other at the outset. As shown below, symmetry considerations reduce the $q-1$ equations to two. Even so, it is not at all easy to solve these equations, even numerically (Dickinson 1984).

Our approach is an analytic one which is valid for the $q$-state Potts model in the limit in which $q-2$ is small. Our principal result is that there is, in fact, a wetting of the interface between ordered phases by the disordered phase as the temperature approaches the bulk transition temperature $T_{0}$. Given that such an interfacial wetting occurs, it is believed that effective one-density theories should yield the correct thermodynamic singularities associated with the interfacial wetting transition (Lipowsky 1984). These theories yield a width of the disordered region which diverges like $\ln \left(T_{0}-T\right)$ (Widom 1978) and an excess surface entropy with the same divergence. Our explicit solution of the coupled equations which depend on all densities explicitly confirms this behaviour.

\section{The equations for the profile}

We consider a $q$-state Potts model on a $d$-dimensional hypercubic lattice governed by the Hamiltonian

$$
H=-J \sum_{\lambda=1}^{q} \sum_{\langle i j\rangle} c_{i}^{\lambda} c_{j}^{\lambda}
$$

where $\lambda$ can take on $q$ values and the second sum is over nearest-neighbour pairs. The variable $c_{i}^{\lambda}$ on the $i$ th site is equal to unity if the Potts spin on that site is in the state $\lambda$ and is zero otherwise. Let the ensemble average of $c_{i}^{\lambda}$ be denoted by $n_{i}^{\lambda}$ which must satisfy the constraint

$$
\sum_{\lambda} n_{i}^{\lambda}=1 \quad \forall i
$$

Then, within mean-field theory, the $n_{i}^{\lambda}$ minimise the free energy functional (Mittag and Stephen 1974)

$$
\beta F=-\beta J \sum_{\lambda} \sum_{\langle i j\rangle} n_{i}^{\lambda} n_{j}^{\lambda}+\sum_{\lambda} \sum_{i} n_{i}^{\lambda} \log n_{i}^{\lambda}
$$

where $\beta$ is the inverse temperature. In the situation of interest to us, a $(d-$ 1)-dimensional planar interface between two ordered phases, the $n_{i}^{\lambda}$ vary in one direction only so that (2) may be simplified to

$$
\beta F / \mathscr{A} \equiv-\beta \sum_{\lambda} \sum_{k} n_{k}^{\lambda}\left(d n_{k}^{\lambda}+\frac{1}{2} \Delta n_{k}^{\lambda}\right)+\sum_{\lambda} \sum_{k} n_{k}^{\lambda} \log n_{k}^{\lambda}
$$

where $A$ is the area of a hyperplane, $k$ the index of such planes, and $\Delta$ the second difference operator:

$$
\Delta X_{k} \equiv\left(X_{k+1}-2 X_{k}+X_{k-1}\right) .
$$

Of the $q$ densities $n_{k}^{\lambda}$ on each plane $k$, only two are independent. They can be taken to be $n_{k}^{\mathrm{A}}$ and $n_{k}^{\mathrm{B}}$. By symmetry, all the other $n_{k}^{\lambda}$ are equal and can be obtained from the constraint $(2)$ as

$$
n_{k}^{\lambda}=\left(1-n_{k}^{\mathrm{A}}-n_{k}^{\mathrm{B}}\right) /(q-2) \quad \lambda \neq \mathrm{A}, \mathrm{B} .
$$


Instead of $n_{k}^{\mathrm{A}}$ and $n_{k}^{\mathrm{B}}$ we use the two independent variables

$$
S_{k}=\frac{1}{2}\left(n_{k}^{\mathrm{A}}+n_{k}^{\mathrm{B}}\right)
$$

and

$$
D_{k}=n_{k}^{\mathrm{A}}-n_{k}^{\mathrm{B}}
$$

so that

$$
n_{k}^{\lambda}=\left(1-2 S_{k}\right) /(q-2) \quad \lambda \neq \mathrm{A}, \mathrm{B} .
$$

In terms of these variables, the free energy functional of (4) can be written as

$$
\begin{gathered}
\beta F / \mathscr{A}=\sum_{k} f\left(\left\{S_{k}\right\},\left\{D_{k}\right\}\right) \\
f=-d \beta J\left(2 S_{k}^{2}+\frac{1}{2} D_{k}^{2}+\frac{\left(1-2 S_{k}\right)^{2}}{(q-2)}\right)-\beta J\left(S_{k} \Delta S_{k}+\frac{1}{4} D_{k} \Delta D_{k}\right)+S_{k} \log \left(S_{k}^{2}-\frac{D_{k}^{2}}{4}\right) \\
+\frac{D_{k}}{2} \log \left(\frac{S_{k}+\frac{1}{2} D_{k}}{S_{k}-\frac{1}{2} D_{k}}\right)+\left(1-2 S_{k}\right) \log \left(\frac{1-2 S_{k}}{q-2}\right)+\beta J \frac{1}{q-2}\left(1-2 S_{k}\right) \Delta S_{k} .
\end{gathered}
$$

Variation with respect to the $S_{k}$ and $D_{k}$ leads to the equations

$$
q \beta J \Delta S_{k}=-2 d \beta J\left(S_{k} q-1\right)-(q-2) \log \left(\frac{1-2 S_{k}}{q-2}\right)+\frac{q-2}{2} \log \left(S_{k}^{2}-D_{k}^{2} / 4\right)
$$

and

$$
\beta J \Delta D_{k}=-2 d \beta J D_{k}+\log \left(\frac{S_{k}+D_{k} / 2}{S_{k}-D_{k} / 2}\right) .
$$

These equations always admit the uniform disordered solution

$$
\begin{array}{ll}
S_{k}=1 / q & \forall k \\
D_{k}=0 & \forall k
\end{array}
$$

corresponding to $n_{k}^{\lambda}=1 / q$ for all $k$ and $\lambda$. Below the transition temperature given by

$$
2 d J \beta_{0}=2 \frac{(q-1)}{(q-2)} \log (q-1)
$$

there is another uniform solution (which minimises $f$ ) in which only one colour, A say, is different from the others so that $n_{k}^{\mathrm{B}}$ is the same as $n_{k}^{\lambda}$ for $\lambda$ unequal to A. From the definition of (7) and the value of $n_{k}^{\lambda}$ given in (8) we find that the uniform values of $S_{k}$ and $D_{k}$, which for this solution we denote $S_{\mathrm{B}}$ and $D_{\mathrm{B}}$, are related by

$$
S_{\mathrm{B}}-\frac{D_{\mathrm{B}}}{2}=\frac{1-2 S_{\mathrm{B}}}{q-2} \text {. }
$$

Knowing this, one obtains the solution of (11) and (12) from the solution of

$$
\frac{2\left(S_{\mathrm{B}} q-1\right)}{q-2}=\frac{1}{2 d \beta J} \log \left(\frac{2 S_{\mathrm{B}} q-2 S_{\mathrm{B}}-1}{1-2 S_{\mathrm{B}}}\right)
$$

and

$$
\frac{D_{\mathrm{B}}}{2}=\frac{S_{\mathrm{B}} q-1}{q-2}
$$


At the transition temperature, the explicit solution is

$$
\begin{array}{ll}
S_{\mathrm{B}}=\left(q^{2}-2 q+2\right) / 2 q(q-1) & T=T_{0} \\
D_{\mathrm{B}}=(q-2) /(q-1) & T=T_{0}
\end{array}
$$

corresponding to

$$
\begin{aligned}
& n_{k}^{\mathrm{A}}=(q-1) / q \\
& n_{k}^{\lambda}=1 / q(q-1) \quad \lambda \neq \mathrm{A} .
\end{aligned}
$$

Comparison with (13) and (14) shows the first-order nature of the transition at $T_{0}$.

To determine the surface tension $\sigma_{\mathrm{AB}}(T)$, we need to solve equations (11) and (12) subject to the boundary conditions

$$
\begin{array}{ll}
S_{k} \rightarrow S_{\mathrm{B}} & k \rightarrow \pm \infty \\
D_{k} \rightarrow \pm D_{\mathrm{B}} & k \rightarrow \pm \infty .
\end{array}
$$

We denote these solutions $\hat{S}_{k}$ and $\hat{D}_{k}$. Then $\sigma_{\mathrm{AB}}$ is obtained from

$$
\beta \sigma_{\mathrm{AB}}=\sum_{k} f\left(\left\{\hat{S}_{k}\right\},\left\{\hat{D}_{k}\right\}\right)-f\left(\left\{S_{\mathrm{B}}\right\},\left\{D_{\mathrm{B}}\right\}\right)
$$

with $f$ given by (10). A measure of the amount of the disordered phase absorbed at the interface is provided by

$$
\begin{aligned}
W & \equiv \sum_{\lambda \neq A, \mathrm{~B}} \sum_{k}\left(n_{k}^{\lambda}\left(\hat{S}_{k}, \hat{D}_{k}\right)-n_{k}^{\lambda}\left(S_{\mathrm{B}}, D_{\mathrm{B}}\right)\right) \\
& =2 \sum_{k}\left(S_{\mathrm{B}}-\hat{S}_{k}\right) .
\end{aligned}
$$

It is not an easy task to solve (11) and (12) with the boundary conditions (23) and (24), even numerically. There is almost certainly no unique solution to the equations with such boundary conditions but rather a set of solutions corresponding to a series of local minima (and maxima) of (25) and one global minimum. If several of the local minima are almost degenerate with the global one, the problem of determining the latter is considerable. We avoid this problem in the next section by solving the equations analytically in the neighbourhood of $q$ equal to 2 and for temperatures $T$ close to $T_{0}$ (in particular for $T_{0}-T \sim(q-2)^{2}$ ). This will allow us to give a complete description of the profile of the interface.

\section{The profile for $q$ close to 2}

For $q$ close to 2 , and $T$ close to $T_{0}$, one can simplify equations (11) and (12) which give the profile. To do so, let us define $\psi$ by

$$
\psi=q-2
$$

and $\varepsilon$ by

$$
2 d \beta J-2 d \beta_{0} J=\varepsilon .
$$

Thus $\varepsilon$ gives a measure of the difference $T_{0}-T$. If we define $\mu$ as

$$
\mu=\varepsilon / \psi^{2}
$$


then we shall consider $\mu$ to be of order unity. One can expand $2 d \beta_{0} J$ given by (15) in powers of $\psi$ and one finds

$$
2 d \beta J=2 d \beta_{0} J+\mu \psi^{2}=2+\psi-\frac{1}{3} \psi^{2}+\frac{1}{6} \psi^{3}+\mu \psi^{2}+\ldots
$$

Let us assume that

$$
\begin{aligned}
& D_{k}=\mathrm{O}(\psi) \\
& S_{k}=\frac{1}{2}-\frac{1}{4} \psi+\frac{1}{4} u_{k}
\end{aligned}
$$

where

$$
u_{k}=\mathrm{O}\left(\psi^{2}\right) \text {. }
$$

The idea of our solution is a self-consistent one. First we assume (31), (32) and (33), i.e. that $D_{k}$ is of order $\psi$ along the profile and that the $k$ dependence of $S_{k}$ is of order $\psi^{2}$. Then we simplify equations (11) and (12) using this assumption and find a solution which is consistent with it.

Using (27) and (29)-(33) one can expand the right-hand side of equations (11) and (12) up to the third order in $\psi$. It turns out that the zeroth, first and second orders vanish and just the third order remains. The result is

$$
\begin{aligned}
& \beta J\left(D_{k+1}+D_{k-1}-2 D_{k}\right)=\left(\frac{5}{6} \psi^{2}-\mu \psi^{2}-u_{k}\right) D_{k}+\frac{2}{3} D_{k}^{3} \\
& \frac{1}{2} q \beta J\left(u_{k+1}+u_{k-1}-2 u_{k}\right)=\frac{1}{4} \psi^{3}-\psi u_{k}-\psi D_{k}^{2}+u_{k}^{2} / \psi .
\end{aligned}
$$

We seek a solution such that $u_{k} \rightarrow u_{\mathrm{B}}$ and $D_{k} \rightarrow \pm D_{\mathrm{B}}$ when $k \rightarrow \pm \infty$ where $u_{\mathrm{B}}$ and $D_{\mathrm{B}}$ are given by

$$
\begin{aligned}
& u_{\mathrm{B}}=\frac{1}{4}\left[5+(1+24 \mu)^{1 / 2}\right] \psi^{2}+\text { higher-order terms } \\
& D_{\mathrm{B}}=\frac{1}{4}\left[3+(1+24 \mu)^{1 / 2}\right] \psi+\text { higher-order terms. }
\end{aligned}
$$

For small $\psi$, the right-hand sides of (34) and (35) are of order $\psi^{3}$ whereas the left-hand side of (34) is a priori of order $\psi$ and of (35) of order $\psi^{2}$. The only way of solving these equations is to look for a solution which varies slowly with $k$. By inspection (34) and (35) have a solution of the form

$$
\begin{aligned}
& D_{k}=\psi G\left(\frac{1}{(\beta J)^{1 / 2}} \psi k\right)+\text { higher-order terms } \\
& u_{k}=\psi^{2} H\left(\frac{1}{(\beta J)^{1 / 2}} \psi k\right)+\text { higher-order terms }
\end{aligned}
$$

where the functions $G(t)$ and $H(t)$ satisfy the following two equations

$$
\begin{aligned}
& \mathrm{d}^{2} G / \mathrm{d} t^{2}=\left(\frac{5}{6}-\mu-H\right) G+\frac{2}{3} G^{3} \\
& \frac{1}{2} \psi q \mathrm{~d}^{2} H / \mathrm{d} t^{2}=\frac{1}{4}-H-G^{2}+H^{2} .
\end{aligned}
$$

Here one should notice the fact which makes the problem soluble: the term which contains $\mathrm{d}^{2} H / \mathrm{d} t^{2}$ in (41) is a higher-order term (since it is multiplied by $\psi$ ) and therefore $(41)$ can be replaced by

$$
\frac{1}{4}-H-G^{2}+H^{2}=0 \text {. }
$$

Together with the boundary conditions (36) and (37), this yields

$$
H=\frac{1}{2}+|G| \text {. }
$$


Using (43) and (40), one sees that the problem reduces to finding the solution of the differential equation for $G$

$$
\mathrm{d}^{2} G / \mathrm{d} t^{2}=\left(\frac{1}{3}-\mu-|G|\right) G+\frac{2}{3} G^{3}
$$

which tends to $\pm \frac{1}{4}\left[3+(1+24 \mu)^{1 / 2}\right]$ when $t \rightarrow \pm \infty$.

One can integrate this equation (44) and obtain the constant of integration from the fact that $\mathrm{d} G / \mathrm{d} t$ vanishes as $t \rightarrow \pm \infty$. This leads to

$(\mathrm{d} G / \mathrm{d} t)^{2}=\frac{1}{3} G^{2}(1-|G|)^{2}-\mu G^{2}+\frac{1}{96}\left[-1+60 \mu+72 \mu^{2}+(1+24 \mu)^{3 / 2}\right]$.

With the solution of $(45)$, one knows the profile

$$
\begin{aligned}
& D_{k}=\psi G\left(\frac{1}{(\beta J)^{1 / 2}} \psi k\right) \\
& S_{k}=\frac{1}{2}-\frac{1}{4} \psi+\frac{1}{8} \psi^{2}+\frac{1}{4} \psi^{2}\left|G\left(\frac{1}{(\beta J)^{1 / 2}} \psi k\right)\right| .
\end{aligned}
$$

For arbitrary $\mu$, one can solve (45) numerically or try to express its solution in terms of tabulated functions. However, to study the critical behaviour $\left(\left(T_{0}-T\right) \ll 1\right)$ one needs only to consider the limit $\mu \rightarrow 0$. For $\mu$ small, the function $G$ varies from $-(1+3 \mu)$ to $1+3 \mu$ when $t$ goes from $-\infty$ to $+\infty$. The critical behaviour will be dominated by the length $L$ along which $G$ remains close to 0 . For $|G(t)|$ small and $\mu$ small, (45) can be simplified to

$$
(\mathrm{d} G / \mathrm{d} t)^{2}=\frac{1}{3} G^{2}+\mu
$$

which has the solution

$$
G(t)=(3 \mu)^{1 / 2} \sinh (t / \sqrt{3}) .
$$

Therefore the length $L$ over which $|G(t)|$ is small diverges like

$$
L \simeq-\sqrt{3} \log \mu \quad \text { for } \mu \rightarrow 0 .
$$

We can now estimate $W$, the amount of disordered phase absorbed at the interface. From (26) and (47), one has for $\psi$ small

$$
W \simeq \frac{\psi^{2}}{2} \sum_{k}\left[\frac{3+(1+24 \mu)^{1 / 2}}{4}-\left|G\left(\frac{1}{(\beta J)^{1 / 2}} \psi k\right)\right|\right] .
$$

For $\mu$ small the sum is dominated by the region where $G$ is close to 0 :

$$
W \simeq \frac{\psi^{2}}{2}\left(\frac{(\beta J)^{1 / 2}}{\psi} L\right) \simeq-\frac{\sqrt{3} \psi}{2 \sqrt{d}} \log \mu
$$

where we have used $d \beta_{0} J=1$ for $q=2$.

Thus in terms of the variables $q$ and $T$, one finds that for $q-2$ small and $T_{0}-T \ll$ $(q-2)^{2}$ :

$$
W \simeq-\frac{\sqrt{3}}{2 \sqrt{d}}(q-2) \log \left(\frac{T_{0}-T}{(q-2)^{2} T_{0}}\right) .
$$

Thus, interfacial wetting does indeed take place as the temperature approaches $T_{0}$. simple:

At the bulk critical temperature itself $(\mu=0)$, the solution of $(45)$ is particularly

$$
\tilde{G}(t)= \pm \frac{1}{\exp \left[ \pm\left(t-t_{0}\right) / \sqrt{3}\right]+1}
$$


$t_{0}$ is an arbitrary constant of integration which reflects the fact that the location of the interface is arbitrary. The interface between phases A and B disassociates into two interfaces, between $A$ and the disordered phase, and between the disordered phase and $B$, with profiles given by (54).

Equation (44) can also be used to describe finite-size effects. If one wants to describe an interface $\mathrm{AB}$ between two planes at distance $\mathscr{L}$, then the boundary condition $\mathrm{d} G / \mathrm{d} t \rightarrow 0$ for $t \rightarrow \pm \infty$ has to be replaced by boundary conditions at $t= \pm \mathscr{L}(\beta J)^{1 / 2} / 2 \psi$.

\section{The surface tension for $q$ close to 2}

We have seen that the surface tension $\sigma_{\mathrm{AB}}$ is given by (25) and (10). If we expand $\sigma_{\mathrm{AB}}$ up to the fourth order in $\psi$ (which is the leading order), and use the equations (27)-(35), we find

$\beta \sigma_{\mathrm{AB}}(T)=\sum_{k}\left(-\frac{1}{12}\left(D_{k}^{4}-D_{\mathrm{B}}^{4}\right)+\frac{1}{16} \psi^{2}\left(u_{k}-u_{\mathrm{B}}\right)-\frac{1}{8}\left(u_{k}^{2}-u_{\mathrm{B}}^{2}\right)+\frac{1}{12} \psi^{-2}\left(u_{k}^{3}-u_{\mathrm{B}}^{3}\right)\right)$

where $u_{\mathrm{B}}$ and $D_{\mathrm{B}}$ are the bulk values given by (36) and (37) and $u_{k}$ and $D_{k}$ are the solutions of (34) and (35). At the bulk transition temperature

$$
\begin{aligned}
\beta \sigma_{\mathrm{AB}}\left(T_{0}\right) & =\frac{\psi^{4}}{12}\left(\sum_{k=-\infty}^{\infty}\left|\tilde{G}^{3}\left[\psi k /(\beta J)^{1 / 2}\right]\right|-\tilde{G}^{4}\left[\psi k /(\beta J)^{1 / 2}\right]\right) 2 \\
& =\frac{\psi^{3}}{12}(\beta J)^{1 / 2} \int_{-\infty}^{\infty}\left[\left|\tilde{G}^{3}(t)\right|-\tilde{G}^{4}(t)\right] 2 \mathrm{~d} t
\end{aligned}
$$

where $\tilde{G}$ is given by (54).

The last factor of 2 comes from the fact that there are two interfaces between phases $A$ and $B$ which are an infinite distance apart. Clearly the surface tension $\sigma$ between the ordered and disordered phases, which is defined only at $T_{0}$, is given by

$$
\sigma=\frac{1}{2} \sigma_{\mathrm{AB}}\left(T_{0}\right) \text {. }
$$

For $q-2$ small, the result of the expression (57) can be written:

$$
\beta \sigma_{\mathrm{AB}}\left(T_{0}\right) \simeq \frac{(\beta J)^{1 / 2} \psi^{3}}{6 \sqrt{3}} \simeq \frac{(q-2)^{3}}{6(3 d)^{1 / 2}}
$$

One can also calculate $\sigma_{\mathrm{AB}}(T)$ for $T<T_{0}$. For small $\mu$ one finds that the main contribution to the difference $\sigma_{\mathrm{AB}}(T)-\sigma_{\mathrm{AB}}\left(T_{0}\right)$ comes from the length $L$ of the intervening disordered region

$$
\beta\left[\sigma_{\mathrm{AB}}(T)-\sigma_{\mathrm{AB}}\left(T_{0}\right)\right]=\left(\frac{1}{4} \mu \psi^{4}\right)(-\sqrt{3} \log \mu)\left[(\beta J)^{1 / 2} / \psi\right] .
$$

In this equation, $\frac{1}{4} \mu \psi^{4}$ is the difference in free energy between the ordered and disordered phases. It is linear in $T-T_{0}$ because the transition is first order. The remaining factor is the length $L$ of equation (50) expressed in units of the lattice spacing. Expressing this result in terms of $T_{0}-T$ and $q-2$ and again using $(\beta J)^{1 / 2}=$ $d^{-1 / 2}$ for $q=2$ one obtains

$$
\beta\left(\sigma_{\mathrm{AB}}(T)-\sigma_{\mathrm{AB}}\left(T_{0}\right)\right) \sim-\left(\frac{3}{4 d}\right)^{1 / 2}(q-2)\left(\frac{T_{0}-T}{T_{0}}\right) \log \left(\frac{\left(T_{0}-T\right)}{(q-2)^{2}}\right) .
$$


Equations (53) and (61) are the main results of this work. They are valid for $(q-2) \ll 1$ and $0<T_{0}-T \ll(q-2)^{2}$. One can, of course, calculate $W$ and $\sigma_{\mathrm{AB}}(T)$ for $(q-2)$ small and $\left(T_{0}-T\right) /(q-2)^{2}$ not small from the solution of $(45)$.

\section{Discussion}

We have seen that, for $q$ close to 2 and within mean-field theory, there is a wetting of the interface between two ordered phases of the $q$-state Potts model by the disordered phase as $T$ approaches $T_{0}$, the temperature at which all three phases are in coexistence. The excess interfacial absorbtion $W$ diverges, according to (53), $\operatorname{like} \log \left(T_{0}-T\right)$. In addition, equation (61) for the surface tension shows that the excess surface entropy, $-\partial \sigma_{\mathrm{AB}} / \partial T$, also diverges logarithmically. That these divergences are intimately related is easily seen. For temperatures near $T_{0}$, the thickness $L$ of the disordered region, which intrudes between the two ordered phases, is large and is directly proportional to $W$. Further, the surface tension $\sigma_{\mathrm{AB}}$ varies near $T_{0}$ as $L$ multiplied by the difference of the free energy per unit volume of the ordered and disordered phases at the same temperature. The latter quantity is, of course, well defined in mean-field theory but, even more generally, it can be defined as the continuation of the disordered free energy in the vicinity of $T_{0}$ (we ignore the possibility of essential singularities at a first-order transition). When the bulk transition of the Potts model is first order, this free energy difference is linear in $T_{0}-T$ from which follows the proportionality between the excess absorbtion and excess entropy.

In addition to solving equations (11) and (12) analytically near $q=2$, we have also tried to solve them numerically for larger values $(q=3,10,20)$. This was difficult because the algorithm we used converged very slowly. Our numerical results indicated that there is an interfacial wetting for these larger values of $q$. We were not able to extract from our data the form of the divergence of $W$ or the singular part of $\sigma_{\mathrm{AB}}$ as $T$ approaches $T_{0}$. However, as we have seen, explicit solution of the many-density theory for $q$ near 2 yields the same $q$-independent singularities as obtained from one-component theories which, by their construction, are independent of $q$ and all symmetries (Widom 1977). It is reasonable to expect, therefore, that the results of one-component theories are applicable to the larger values of $q$ as well. Further support for this view derives from the work of Kikuchi and Cahn (1980) which provides an example of an interfacial wetting in a system with more than one component which does not have Potts symmetry but which does display the same singular behaviour as found above.

Lastly we note that the existence of the interfacial wetting could have been anticipated by the use of the sharp-kink approximation in which the smooth continuous variation of all densities through the interface is ignored and the minimum of the surface tension, (25), is sought within the following subspace:

$$
\begin{aligned}
n_{k}^{\lambda} & =n_{\mathrm{A}}^{\lambda} & & k \leqslant 0 \\
& =n_{\text {DIS }} & & 0<k \leqslant Z \\
& =n_{\mathrm{B}}^{\lambda} & & Z<k
\end{aligned}
$$

where $n_{\mathrm{A}}^{\lambda}, n_{\mathrm{B}}^{\lambda}$ and $n_{\mathrm{DIS}}$ are the values of $n_{k}^{\lambda}$ appropriate in the $\mathrm{A}, \mathrm{B}$ and disordered phases at $T_{0}$. In this approximation, $\sigma_{\mathrm{AB}}$ at $T_{0}$ depends only on the thickness of the disordered phase $Z$. Using this ansatz and the fact that all phases are in coexistence, 
we easily obtain

$$
\sigma_{\mathrm{AB}}(Z)-\sigma_{\mathrm{AB}}(0)=-J \sum_{\lambda}\left(n_{\mathrm{A}}^{\lambda}-n_{\mathrm{DIS}}\right)\left(n_{\mathrm{DIS}}-n_{\mathrm{B}}^{\lambda}\right) \quad Z \geqslant 1 \text {. }
$$

As $n_{\lambda^{\prime}}=(q-1) / q$ for $\lambda=\lambda^{\prime}$ and $1 / q(q-1)$ for $\lambda \neq \lambda^{\prime}$ and $n_{\mathrm{DIS}}=1 / q,(63)$ yields

$$
\sigma_{\mathrm{AB}}(Z)-\sigma_{\mathrm{AB}}(0)=-\frac{J(q-2)^{2}}{q(q-1)^{2}} \quad Z \geqslant 1 .
$$

Thus, within this approximation, a layer of disordered phase of any non-zero thickness is favourable over no such layer but $\sigma_{\mathrm{AB}}$ is otherwise independent of $Z$. In the exact solution of the mean-field equations, the densities $n_{k}^{\lambda}$ do not change abruptly, but exponentially ( $\mathrm{cf}(54)$ ). This causes a repulsion between the kinks which in turn causes $\sigma_{\mathrm{AB}}(Z)$ to decrease exponentially with $Z$. Hence an infinite value of $Z$ minimises $\sigma_{\mathrm{AB}}$ which corresponds to a wetted interface.

The value of the interfacial tension at $T_{0}$ in the sharp-kink approximation is

$$
\frac{\sigma_{\mathrm{AB}}}{J}=\frac{(q-2)^{2}}{q(q-1)} \quad \text { (sharp kink) }
$$

which is to be compared with the analytic result of $(59)$ valid for $(q-2)$ small

$$
\frac{\sigma_{\mathrm{AB}}}{J}=\frac{1}{6}\left(\frac{d}{3}\right)^{1 / 2}(q-2)^{3} \text {. }
$$

The latter is smaller than the former by a factor of $\sqrt{d}(q-2)$ which has been assumed to be small. The approximation overestimates the surface tension precisely because the kinks are not sharp but vary on the length scale $1 /[\sqrt{d}(q-2)]$ which is assumed to be large. However for large $q$ the kinks do become sharp so that the approximate expression (65) should become increasingly accurate.

Our results may have some experimental relevance. As we noted earlier, an A/B interface cannot be wetted by another ordered phase. Similarly, the interface between the disordered phase and one ordered phase cannot be wetted by another ordered phase. From this we surmise that in a binary-liquid mixture in which the two fluids are very similar, neither vapour-liquid interface will be wet by the other fluid. Rather, the vapour will wet the liquid/liquid interface. The first statement can be checked experimentally rather easily. The second cannot because gravity prevents the vapour from intervening between the liquids. It might be checked, however, in a threedimensional realisation of the three-state Potts model (Mukamel et al 1977, Barbara et al 1978). This consists of a Heisenberg ferromagnet in a crystal with a strong anisotropy which favours the six directions in the set $\leqslant 100 \geqslant$. A magnetic field along the (111) direction favours the three directions $(100),(010)$ and $(001)$ resulting in a three-state Potts symmetry. Quite generally, there will be domains of the different directions of magnetisation. On heating to $T_{0}$, the paramagnetic phase is predicted to wet the interfaces between these domains, an effect which should be readily observable.

\section{Acknowledgments}

We would like to thank $\mathbf{J}$ Bricmont, L de Seze and J L Lebowitz for several illuminating discussions. One of us (MS) would like to thank the members of the Service de Physique Théorique, CEN-Saclay, for the warm hospitality extended to him. This 
work was supported in part by the National Science Foundation under grant no DMR 83-19301.

\section{References}

Barbara B, Rossignol M F and Bak P 1978 J. Phys. C: Solid State Phys. 11 L183

Dickinson H 1984 PhD Thesis Southampton University

Forgacs G, Orland H and Schick M 1986 Phys. Rev. B 3395

Frenken J W M and van der Veen J F 1985 Phys. Rev. Lett. 54134

Huse D A, Szpilka A M and Fisher M E 1983 Physica 121A 363

Kikuchi R and Cahn J W 1980 Phys. Rev. B 211893

Lipowsky R 1984 J. Appl. Phys. 552485

Mittag L and Stephen M J 1974 J. Phys. A: Math. Gen. 7 L109

Mukamel D, Fisher M E and Domany E 1976 Phys. Rev. Lett. 37565

Selke W 1984 Surf. Sci. 144176

Sullivan D E and Telo da Gama M M 1985 in Fluid Interfacial Phenomena ed C A Croxton (New York: Wiley) Tarazona P, Telo da Gama M M and Evans R 1983 Mol. Phys. 49283

Widom B 1977 Statistical Mechanics and Statistical Methods in Theory and Application ed U Landman (New York: Plenum) p 33

1978 J. Chem. Phys. 683878

Yeomans J and Derrida B 1985 J. Phys. A: Math. Gen. 182343 